\title{
Defect-induced magnetism in chemically synthesized nanoscale sheets of MgO
}

\author{
Ben M. Maoz, ${ }^{1}$ Einat Tirosh, ${ }^{1}$ Maya Bar Sadan, ${ }^{2}$ and Gil Markovich ${ }^{1, *}$ \\ ${ }^{1}$ School of Chemistry, Raymond and Beverly Sackler Faculty of Exact Science, Tel Aviv University, Tel Aviv IL-69978, Israel \\ ${ }^{2}$ Institute of Solid State Research, Ernst Ruska-Centre for Microscopy and Spectroscopy with Electrons-Research Centre Jülich GmbH, \\ D-52425 Jülich, Gernmany \\ (Received 24 February 2011; published 6 April 2011)
}

\begin{abstract}
Highly defective $\mathrm{MgO}$ nanosheets were prepared by a colloidal synthesis and exhibited low-temperature ferromagnetism which was significantly larger that the magnetization potentially obtainable from the low transition-metal impurity concentration. Electron paramagnetic resonance experiments confirmed that the magnetization did not significantly involve impurities and that the nanosheets consisted of strongly interacting spin clusters which disappeared upon high-temperature annealing. These spins were concentrated along extended defects, possibly as unpaired electrons trapped at oxygen vacancies.
\end{abstract}

DOI: 10.1103/PhysRevB.83.161201

PACS number(s): 75.30.Hx, 68.55.Ln, 71.55.Gs, 75.70.Cn

Defect-induced magnetism (DIM), which originally concentrated on carbon-based materials, ${ }^{1}$ began receiving substantial attention in the last decade when the interest shifted towards diamagnetic oxides. This was due to theoretical predictions of vacancy-induced ferromagnetism (FM) in simple oxides $^{2-4}$ and the large interest in oxide-based dilute magnetic semiconductors with Curie temperatures above room temperature, where there was much ambiguity regarding the source of the observed ferromagnetism. It received another boost from a finding of ferromagnetism in thin $\mathrm{HfO}_{2}$ films by Coey and coworkers. ${ }^{5}$ Since then, experimental results from many groups claimed the appearance of DIM in several oxides, primarily $\mathrm{ZnO},{ }^{6} \mathrm{HfO}_{2},{ }^{7} \mathrm{CaO},{ }^{8}$ and $\mathrm{MgO} .{ }^{9-13}$

DIM should be highly sensitive to the various defect or impurity characteristics, posing experimental and theoretical difficulties that demand prudence. Experimentally, Abraham et al.$^{14}$ and Garcia et al. ${ }^{15}$ showed the ease with which a ferromagnetic signal is obtained in such oxides due to impurities. The problem of paramagnetic transition-metal ion impurities as a source of magnetism was recently comprehensively studied by Khalid et al. ${ }^{16}$ Much of the previous experimental work claiming to have detected DIM in $\mathrm{MgO}^{9-13}$ as well as other materials ignored the need to precisely determine the paramagnetic metal impurity concentration in the analysis of results. Preparation of samples by deposition in a stainlesssteel apparatus, ${ }^{12}$ using characterization techniques such as $\mathrm{X}$-ray diffraction (XRD) and $\mathrm{x}$-ray photoelectron spectroscopy (XPS) with insufficient sensitivity to low concentrations of impurities, ${ }^{9,13}$ or using less-than ultrapure precursors or not addressing precursor purity ${ }^{10,11}$ may lead to confusion about the source of the observed ferromagnetism. The theory of DIM is also problematic: In the early 70 s large magnetic moments were predicted on neighboring oxygen atoms due to $\mathrm{Mg}$ vacancies. ${ }^{17}$ Elfimov et al. ${ }^{3}$ predicted cation vacancy-induced ferromagnetism using density functional theory (DFT) calculations. Also, further theoretical work attributed the formation of magnetic moments and the resulting FM to spin-polarized holes residing on cation $p$ orbitals either at vacancy or impurity sites. ${ }^{3,18}$ Most DIM studies, both experimental ${ }^{10-13}$ and theoretical, ${ }^{12,19-21}$ suggested that the origin of the observed FM in these oxides is related to cation vacancies or even nitrogen doping in $\mathrm{MgO} .{ }^{22-24}$ Recent theoretical work discussed interdefect coupling mechanisms, ${ }^{9,25-27}$ such as high carrier mobility near interfaces. Very recently, Zunger and coworkers $^{28,29}$ and Droghetti et al. ${ }^{2}$ drew attention to the Achilles' heel of all DFT calculations performed with local approximations of the exchange correlation potentials, such as the local spin density approximation and the generalized gradient approximation. These methods overestimate spin density delocalization, leading to a half-metallic ground state with a relatively strong interdefect ferromagnetic interaction.

In this paper we present results obtained on highly defective $\mathrm{MgO}$ nanosheets (MgO-NSs) prepared by a colloidal synthesis from the ultrapure precursors using several characterization tools. The combination of impurity-concentration measurements, high-resolution transmission electron microscopy (TEM), magnetometry, electron paramagnetic resonance (EPR), and dielectric measurements indicates the appearance of low-temperature FM in the MgO-NSs which is significantly stronger than the largest FM potentially contributed by transition-metal impurity ions and, thus, primarily defect-related.

The MgO-NSs were prepared from high-purity dendritic pieces of distilled $\mathrm{Mg}$, 99.998\% (Sigma-Aldrich), by a colloidal synthesis as described in detail in Ref. 30. In short, the magnesium was transformed into magnesium ethoxide by heating the metal in excess ethanol in a glove box and then the magnesium ethoxide was quickly decomposed at $>570 \mathrm{~K}$ in a high-boiling-point liquid such as oleylamine or hexadecane under a nitrogen or argon atmosphere. Magnetic measurements were conducted using a Quantum Design MPMS XL5 SQUID magnetometer for NS powders made by $\sim 20$ different syntheses. EPR spectra were collected by a Bruker ELEXYS 500 (X band at $9.5 \mathrm{GHz}$ ) spectrometer.

Extreme caution was taken in the preparation of the $\mathrm{MgO}-\mathrm{NS}$ s to minimize contamination. No metal tools were in contact with the samples at any point along the preparation and the glassware used in the experiments was thoroughly cleaned and only used for these preparations. The impurity contents in the MgO-NSs was analyzed using inductively coupled plasma atomic emission spectroscopy and flame atomic absorption, and it was found that the total amount of manganese $(0.04 \mathrm{ppm})$, nickel $(<0.01 \mathrm{ppm})$, iron $(<0.01 \mathrm{ppm})$, and cobalt $(<0.01 \mathrm{ppm})$ was less than $0.1 \mathrm{ppm}$ (by mass). ${ }^{31}$ 


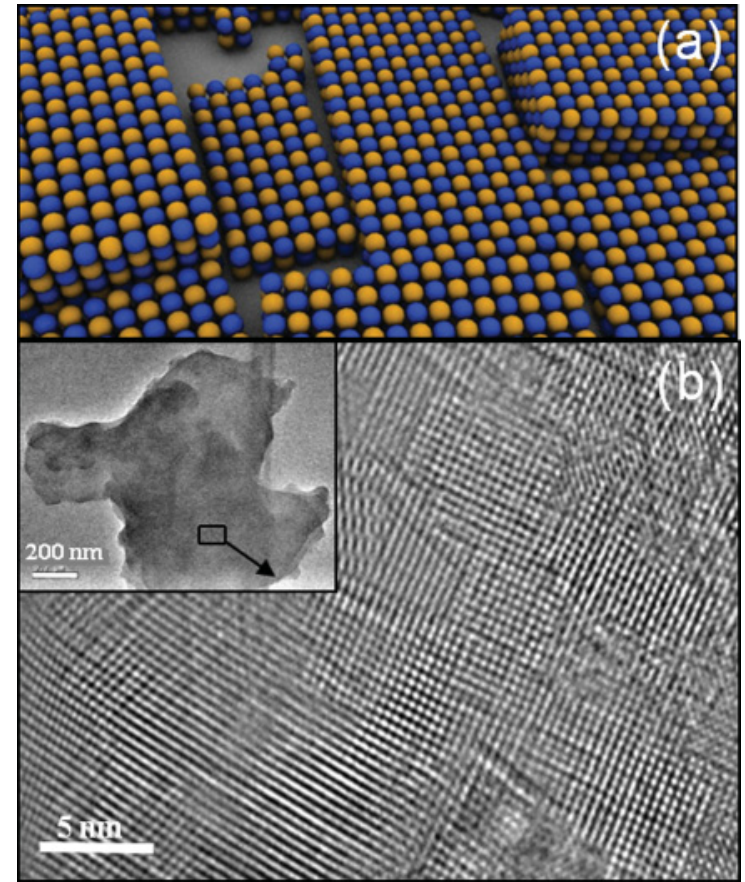

FIG. 1. (Color online) (a) Schematic assembly of an MgO-NS and formation of the dislocations or interfaces. (b) Aberration-corrected high-resolution TEM image of a magnified part of a stack of $\sim 2 \mathrm{MgO}-\mathrm{NSs}$ showing the high density of extended defects.

Figure 1 displays a TEM image of an NS with a magnified high-resolution part of an NS where the high density of extended defects is visible. It was previously estimated that these NSs are about 2 to $3 \mathrm{~nm}$ thick and have a separation between extended defects of the order of $2 \mathrm{~nm}$. This corresponds to an extended defect density of the order of $10^{13} \mathrm{~cm}^{-2}$ (see Fig. 1, and Refs. 30 and 31), which is among the highest obtainable in crystalline materials.

Figure 2(a) displays low-temperature magnetization curves of the precursor Mg-ethoxide, of the as-prepared (AP) $\mathrm{MgO}$ NSs, and of the same sample annealed in air at 423,1023 , and $1373 \mathrm{~K}$ measured by a SQUID magnetometer. The results of $\sim 20$ repeated syntheses were similar qualitatively with some variation $( \pm 30 \%)$ in magnetization values. The standard magnetization curves displayed in Fig. 2(b) show that the precursor powder had a pure diamagnetic response at $4 \mathrm{~K}$ (up to a field of $5 \mathrm{~T}$ ), whereas in Fig. 2(a) it can be seen that the AP NSs exhibit a combination of paramagnetic and ferromagnetic behavior. The inset in the figure shows that the low-temperature hysteresis disappeared around $50 \mathrm{~K}$. Figure 2(c) displays the remnant magnetization $M_{r}(H)$ curves of several samples. The curve is plotted by setting a field value $H$ and nulling the field prior to the magnetization measurement for each field value. Thus, these data distinctively correspond to the ferromagnetic component in the material. The annealed samples, even after air annealing at as-low-as $423 \mathrm{~K}$, lost most of their remnant magnetization. The ferromagnetic component of the AP NS sample had a saturation magnetization of about $3.0 \times 10^{-3} \mathrm{emu} / \mathrm{g}$. It is important to note that MgO-NSs prepared under argon instead of nitrogen exhibited the same FM properties, indicating that the observed DIM is not due to nitrogen doping. If each $\mathrm{Fe}, \mathrm{Co}$, and $\mathrm{Mn}$ impurity would
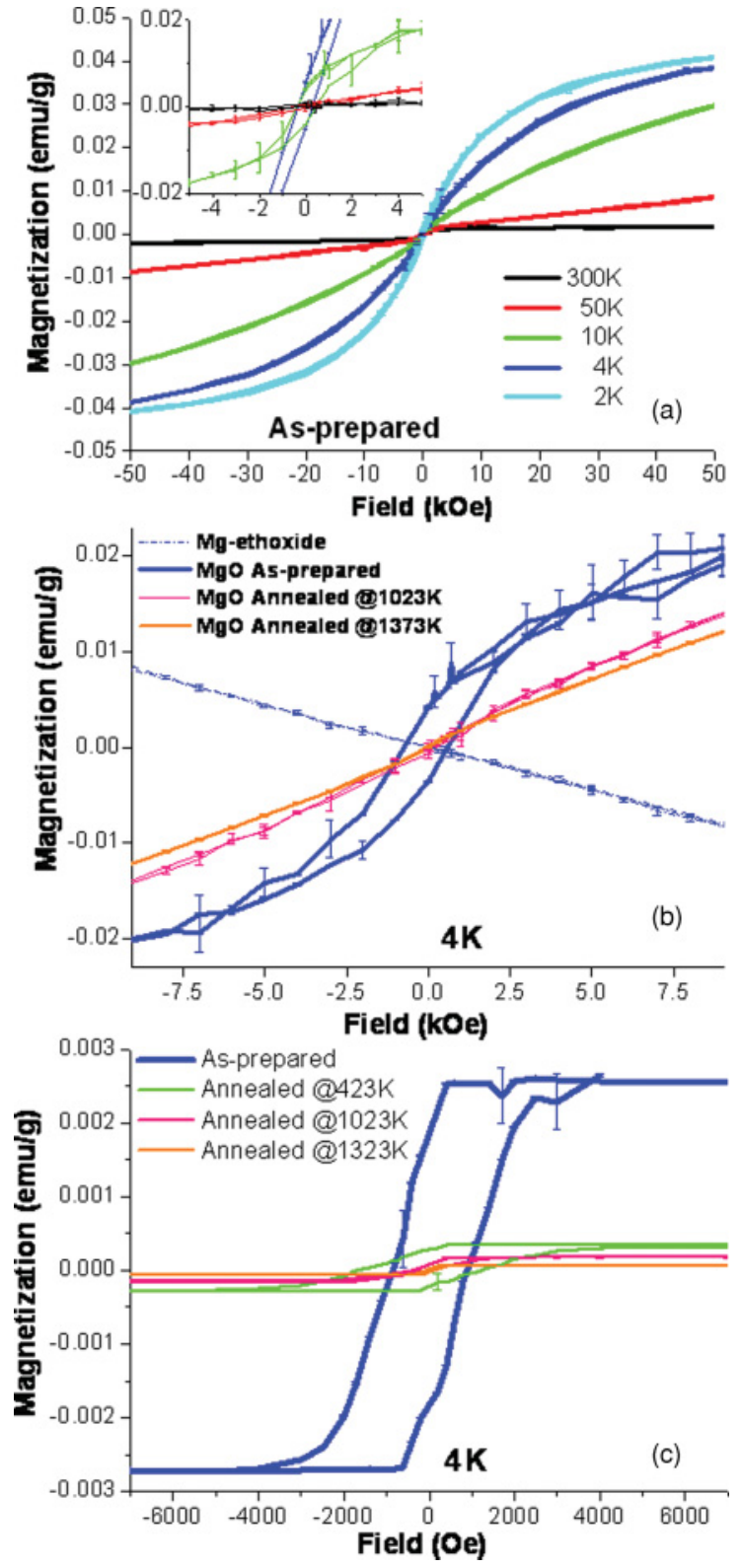

FIG. 2. (Color online) (a) Magnetization curves of the AP sample measured at several temperatures. Inset shows a zoom on the low-field part of the curves. A temperature-independent constant diamagnetic slope was subtracted from all curves. (b) Raw data of 4-K magnetization curves of the precursor Mg-ethoxide, the AP MgO-NSs, and the same sample annealed in air at 1023 and $1373 \mathrm{~K}$. (c) Raw data of 4-K remnant magnetization curves of several samples.

contribute to the saturation magnetization a maximum of $5 \mu_{\mathrm{B}}$, and rounding up the total transition-metal-impurity level to $0.1 \mathrm{ppm}$, the saturation magnetization contributed by the impurities would be $<1 \times 10^{-4} \mathrm{emu} / \mathrm{g}$, which is well below the measured ferromagnetic saturation value and almost three orders of magnitude smaller than the total (paramagnetic + ferromagnetic) magnetization. Thus, it is concluded that the observed FM cannot be explained by impurity-dominated FM.

Long-range interdefect coupling would require some degree of charge delocalization. To study this we have carried out complex dielectric constant measurements of the MgO-NS 


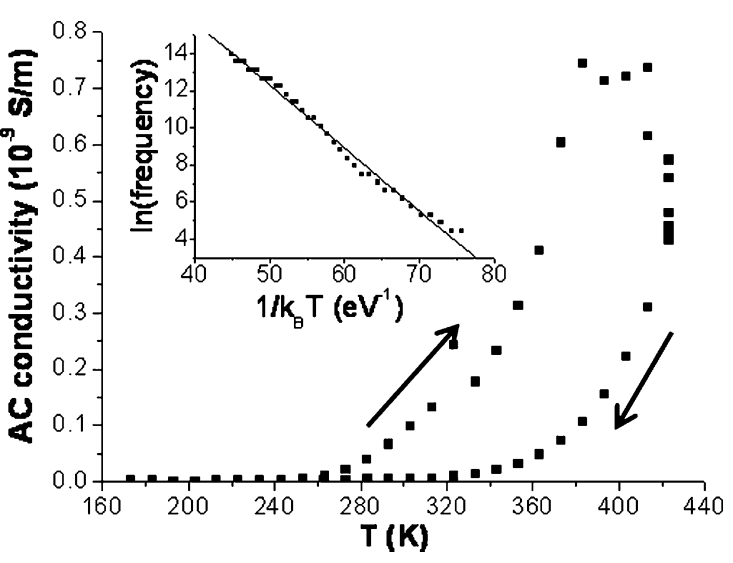

FIG. 3. The AP NSs ac-conductivity dependence on temperature, extracted from the imaginary component of the dielectric constant measured at $1 \mathrm{~Hz}$. The temperature was cycled $170 \rightarrow 420 \rightarrow 170 \mathrm{~K}$ during 2 hours. Inset: A linear fit of the logarithm of the imaginary component peak frequency as a function of reciprocal temperature to extract the charge carrier's trapping barrier (300 meV).

powder. We find that the imaginary part of the dielectric constant is proportional to the conductivity (thus proportional also to mobile charge density). Figure 3 displays the lowfrequency ac-conductivity $(1 \mathrm{~Hz})$ dependence on temperature in a temperature cycling experiment; $170 \rightarrow 420 \rightarrow 170 \mathrm{~K}$. It indicates a strongly thermally activated conductivity, which reaches a maximum conductivity of $\sim 7 \times 10^{-9} \mathrm{~S} / \mathrm{m}$ at $380 \mathrm{~K}$ and is strongly suppressed by annealing at $\sim 420 \mathrm{~K}$, as was the case with the remnant magnetization. A delocalization energy barrier of $\sim 300 \mathrm{meV}$ was estimated from an Arrhenius fit of the temperature dependence of the conductivity-peak frequency (inset of Fig. 3). Thus, it appears that a mild heating for a period of several hours that barely affects the XRD results ${ }^{30}$ was enough to significantly reduce long-range charge mobility in the NSs. This would most probably be related to electrons trapped in dislocations or grain boundaries, as predicted by McKenna and Shluger. ${ }^{32}$ The appearance of FM at relatively low temperatures, where charge mobility is reduced, could be explained by charges trapped at the interfaces or extended defects which may retain a significant degree of delocalization. ${ }^{26,32}$

Figure 4 displays the temperature-dependent EPR spectra of the AP (a), 1023-K (b), and 1323-K (c) annealed NS powders that were previously used for the magnetometry measurements. The first two spectral series shown in Figs. 4(a) and 4(b) do not resemble previous EPR spectra of $\mathrm{MgO}$ with isolated defects. ${ }^{33-35}$ Comparison between the samples shows that the low-field EPR spectra of the AP MgO-NSs possess a large broad peak, conclusively showing that the spins in the system are strongly coupled and form spin clusters, probably at dislocations. ${ }^{36}$ Note that the intensity distribution of this peak further shifts to lower fields with decreasing temperature as more spins become coupled and the interacting spin clusters increase in size. Cooling below $100 \mathrm{~K}$ caused further smearing of the peak and is thus not shown here. Surprisingly, a major part of the coupled spin clusters remained also upon annealing at $1023 \mathrm{~K}$, although shifted to higher field and with a larger fraction of weakly coupled spins around $g \sim 2$. However, upon annealing at $1323 \mathrm{~K}$, where a substantial sintering

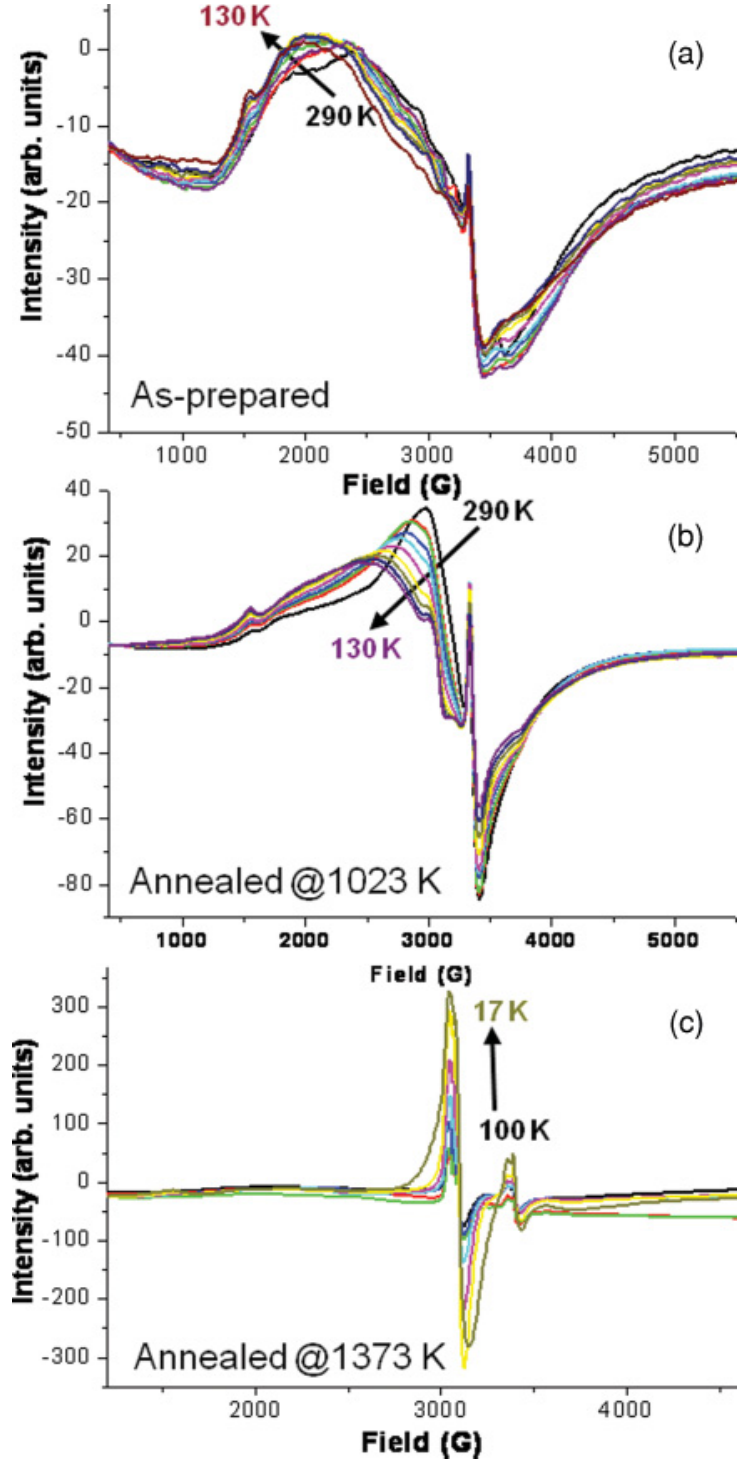

FIG. 4. (Color online) Temperature-dependent EPR X-band spectra of (a) the AP NS powder, (b) the 1023-K, and (c) the 1323-K annealed NS powders. Sample (c) was only measured at low temperatures and is shown in a narrower field range.

process occurred, the defect density was reduced, as seen from the reduction in peak area, and the new split defect signal appeared primarily at $g \sim 1.97$ and $g \sim 2.17$, probably related to trapping of superoxide $\left(\mathrm{O}_{2}^{-}\right)$radicals in the air-annealed $\mathrm{MgO} .33$

The small peak apparent in the spectra at $\sim 1500 \mathrm{G}$ indicates the presence of a low concentration of high-spin $\mathrm{Fe}^{3+}$. Characteristic multiplets of manganese ions were not visible in any of the EPR spectra. Organic radicals can be ruled out as the source of the EPR signal since air annealing at $1023 \mathrm{~K}$ should eliminate all organic material.

Theoretical work has shown that, in order to achieve FM in $\mathrm{MgO}$, the system should have a prohibitively high cation vacancy concentration - of the order of a few percent. For example, Zunger and coworkers calculated a minimum of $4.6 \%$ cation vacancies in $\mathrm{CaO},{ }^{20}$ which is far from the 
thermodynamically allowed concentration. However, as mentioned above, these calculations have overestimated the degree of spin delocalization and it is now realized that even such high point-defect densities would not lead to FM. In addition, all the calculations considered a model of randomly distributed cation vacancies in a bulk oxide lattice ${ }^{12,19-21}$ whereas the present data indicates a different possibility. Since the $\mathrm{MgO}-\mathrm{NSs}$ have a high density of dislocations (Fig. 1, and Ref. 31), we believe that the majority of point defects is concentrated at interfaces, such as along the dislocations or grain boundaries. Recent work supports the concept of strong magnetic interdefect coupling at interfaces. ${ }^{25-27}$

McKenna et al $^{37}$ predicted that vacancies in systems like $\mathrm{MgO}$ tend to segregate to grain boundaries. In these systems, electrons and holes can form delocalized but confined states. A desired long-range interaction may be possible due to the proximity of localized magnetic centers to an extended defect. One dislocation about every $1.5-2 \mathrm{~nm}$ is the estimated density required for achieving DIM at grain boundaries or dislocations, ${ }^{26}$ equivalent to a dislocation density of about $10^{13} \mathrm{~cm}^{-2}$, nicely agreeing with the dislocation density of the NSs in the present work. ${ }^{31}$ Moreover, the high surfaceto-volume ratio of the NSs allows a high density of surface defects. Additional XPS and photoluminescence data, ${ }^{31}$ together with calculations by McKenna and Shluger, ${ }^{36}$ indicate that the most abundant point defects along the interfaces were $\mathrm{F}^{+}$states, which are unpaired electrons trapped at an oxygen vacancy.

In conclusion, this work investigated the phenomenon of low-temperature FM observed in ultra-pure, highly defective $\mathrm{MgO}-\mathrm{NS}$. The $\mathrm{MgO}-\mathrm{NS}$ s were characterized by an extremely high defect density, which is necessary for DIM. Extreme precautions were taken when dealing with contamination and impurity issues, starting from the choice of an ultrapure precursor, using a metal-free environment, and an extensive analysis of the product. The present combination of impurity analysis, observation of simultaneous high extended-defect concentration, relatively high conductivity, low-temperature remnant magnetization, and strongly interacting spin clusters provides strong evidence for the presence of DIM. In contrast to the commonly used randomly distributed cation vacancy model, it is believed that high densities of interface defects at dislocations or grain boundaries are responsible for the DIM effect.

This research was supported by The Israel Science Foundation Grant No. 779/06. The authors are grateful to Lev Weiner for the EPR measurements and to Inna Popov and Yuri Rosenberg for fruitful discussions.
*Corresponding author: gilmar@post.tau.ac.il

${ }^{1}$ P. Esquinazi et al., Phys. Rev. Lett. 91, 227201 (2003).

${ }^{2}$ A. Droghetti, C. D. Pemmaraju, and S. Sanvito, Phys. Rev. B 81, 092403 (2010).

${ }^{3}$ I. S. Elfimov, S. Yunoki, and G. A. Sawatzky, Phys. Rev. Lett. 89, 216403 (2002).

${ }^{4}$ J. Osorio-Guillen, S. Lany, S. V. Barabash, and A. Zunger, Phys. Rev. B 75, 184421 (2007).

${ }^{5}$ M. Venkatesan, C. B. Fitzgerald, and J. M. D. Coey, Nature (London) 430, 630 (2004).

${ }^{6}$ Q. Xu et al., Appl. Phys. Lett. 92, 082508 (2008).

${ }^{7}$ E. Tirosh and G. Markovich, Adv. Mater. 19, 2608 (2007).

${ }^{8}$ D. Gao et al., J. Phys. Chem. C 114, 11703 (2010).

${ }^{9}$ J. I. Beltran, C. Monty, L. Balcells, and C. Martínez-Boubeta, Solid State Comm. 149, 1654 (2009).

${ }^{10}$ J. Hu, Z. Zhang, M. Zhao, H. Qin, and M. Jiang, Appl. Phys. Lett. 93, 192503 (2008).

${ }^{11}$ N. Kumar, D. Sanyal, and A. Sundaresan, Chem. Phys. Lett. 477, 360 (2009).

${ }^{12}$ C. Martínez-Boubeta et al., Phys. Rev. B 82, 024405 (2010).

${ }^{13}$ C. M. Araujo et al., Appl. Phys. Lett. 96, 232505 (2010).

${ }^{14}$ D. W. Abraham, M. M. Frank, and S. Guha, Appl. Phys. Lett. 87, 252502 (2005).

${ }^{15}$ M. A. Garcia et al., J. Appl. Phys. 105, 013925 (2009).

${ }^{16}$ M. Khalid et al., Phys. Rev. B 81, 214414 (2010).

${ }^{17}$ A. M. Stoneham, A. P. Pathak, and R. H. Bartram, J. Phys. C: Solid State Phys. 9, 73 (1976).

${ }^{18}$ C. Das Pemmaraju and S. Sanvito, Phys. Rev. Lett. 94, 217205 (2005).
${ }^{19}$ F. Gao et al., Solid State Comm. 149, 855 (2009).

${ }^{20}$ J. Osorio-Guillen, S. Lany, S. V. Barabash, and A. Zunger, Phys. Rev. Lett. 96, 107203 (2006).

${ }^{21}$ F. Wang et al., Phys. Rev. B 80, 144424 (2009).

${ }^{22}$ I. S. Elfimov et al., Phys. Rev. Lett. 98, 137202 (2007).

${ }^{23}$ V. Pardo and W. E. Pickett, Phys. Rev. B 78, 134427 (2008).

${ }^{24}$ M. Pesci, F. Gallino, C. Di Valentin, and G. Pacchioni, J. Phys. Chem. C 114, 1350 (2009).

${ }^{25}$ S. Gallego, J. I. Beltran, J. Cerda, and M. C. Munoz, J. Phys.: Condens. Matter 17, L451 (2005).

${ }^{26}$ A. M. Stoneham, J. Phys.: Condens. Matter 22, 074211 (2010).

${ }^{27}$ I. de P. R. Moreira, J. C. Wojdel, F. Illas, M. Chiesa, and E. Giamello, Chem. Phys. Lett. 462, 78 (2008).

${ }^{28}$ S. Lany and A. Zunger, Modell. Simul. Mater. Sci. Eng. 17, 084002 (2009).

${ }^{29}$ A. Zunger, S. Lany, and H. Raebiger, Physics 3, 53 (2010).

${ }^{30}$ B. Maoz et al. (unpublished).

${ }^{31}$ See supplemental material at [http://link.aps.org/supplemental/ 10.1103/PhysRevB.83.161201] for further information.

${ }^{32}$ K. P. McKenna and A. L. Shluger, Nat. Mater. 7, 859 (2008).

${ }^{33}$ M. Chiesa, E. Giamello, and M. Che, Chem. Rev. 110, 1320 (2009).

${ }^{34}$ M. Chiesa et al., J. Phys. Chem. B 109, 7314 (2005).

${ }^{35}$ M. Sterrer, O. Diwald, and E. Knozinger, J. Phys. Chem. B 104, 3601 (2000).

${ }^{36}$ K. P. McKenna and A. L. Shluger, Phys. Rev. B 79, 224116 (2009).

${ }^{37}$ K. P. McKenna, P. V. Sushko, and A. L. Shluger, J. Am. Chem. Soc. 129, 8600 (2007). 